\title{
Letter \\ Comments on the study by Taniguchi and coworkers - proving Hippocrates is alive
}

\author{
Nahit Cakar
}

Istanbul Medical Faculty, Anesthesiology and Intensive Care, Capa/Istanbul, Turkey 34093

Corresponding author: Nahit Cakar, cakarn@istanbul.edu.tr

Published: 24 August 2009

Critical Care 2009, 13:415 (doi:10.1186/cc7957)

This article is online at http://ccforum.com/content/13/4/415

(c) 2009 BioMed Central Ltd

See related research by Taniguchi et al., http://ccforum.com/content/13/1/R6, and related commentary by Adigüzel et al., http://ccforum.com/content/13/3/142

I read with interest the article by Taniguchi and coworkers [1] and its accompanying commentary by Adigüzel and colleagues [2] in Critical Care. I have some questions concerning the methodology of the study. Taniguchi and coworkers randomized the postoperative patients to two groups: automated pressure support (PS) mandatory rate ventilation (MRV) and manual PS. In the automated PS MRV group, the patient's expected respiratory rate (RR) was used as a guide to adjust the PS level, employing the algorithm of the Taema-Horus Ventilator ${ }^{\circledR}$ (Air Liquid, France) in MRV mode. However, in the manual PS group the guide for adjusting the PS level was tidal volume/RR (which was kept less than $80 \mathrm{l}$ ), and adjustments were done manually every 30 minutes by intensive care staff.

The study did not compare automated weaning with manual weaning. Rather, it compared automated weaning using a RR target versus manual weaning using tidal a volume/RR ratio target. To justify the conclusions reached by Taniguchi and coworkers and the title of the report, these treatment groups would have needed to differ only in terms of the automated versus manual management component.

Second, they weaned the patients when the PS level decreased to 5 to $7 \mathrm{cmH}_{2} \mathrm{O}$ without conducting a spontaneous breathing trial at the start of the study. This may complicate weaning and prolong the weaning time in this group of patients.

Third, are postoperative patients suitable for such a weaning study? It is likely that whatever protocol you use for weaning, most of them will be weaned without any difficulty in a very short period of time.

To prove that Hippocrates is alive we need fine-tuned studies. If not we may believe that he is alive but it will be unproven.

\section{Authors' response}

\section{Corinne Taniguchi and Carmen SV Barbas}

We read with interest the comments by Cakar about our article [1] and the commentary by Adıgüzel and colleagues in Critical Care [2]. Regarding Cakar's doubts about the methodology of our study that randomized postoperative patients to mandatory rate ventilation or to manual reduction of pressure support, we invite Cakar to observe Figures 5 and 7 of Taniguchi and colleagues study [1] in which the intention to treat analysis showed a mean respiratory rate around $15 \mathrm{bpm}$ and a mean RR/TV (L) around 30 in both groups with no statistical differences between them during the weaning period. According to these results Taniguchi and colleagues actually compared automatic versus manual pressure support reduction in the weaning of postoperative patients as stated in the title of the study.
Regarding the second question about the fact that Taniguchi and colleagues did not perform a spontaneous breathing trial at the initiation of the study the authors would like to clarify that their postoperative patients were sedated and recovering from paralysis at ICU entry, this being the main reason of the absence of spontaneous breathing trial at the beginning of the protocol. Thirdly, our postoperative population consisted of patients that the anesthesiologists decided not to extubate in the recovery room and sent to the ICU for weaning. These postoperative patients are a very good population to test concerning the clinical performance of automatic weaning algorithm. Finally, quoting Adıgüzel, Güngör and Tobin, we also believe that Hippocrates is alive and weaning in Brazil. 


\section{Competing interests}

The author declares that they have no competing interests.

\section{References}

1. Taniguchi C, Eid RC, Saghabi Cilene, Souza R, Silva E, Knobel E, Paes ÂT, Barbas CS: Automatic versus manual pressure support reduction in the weaning of post-operative patients: a randomised controlled trial. Crit Care 2009, 13:R6.

2. Adıgüzel N, Güngör G, Tobin Martin J: Hippocrates is alive and weaning in Brazil. Crit Care 2009, 13:142. 